\title{
QUEEN'S
UNIVERSITY
BELFAST
}

\section{A Data Reduction Pipeline for Gemini-North's Near-infrared Integral Field Spectrometer}

Lemoine-Busserolle, M., Comeau, N., Kielty, C., Klemmer, K., \& Schwamb, M. E. (2019). A Data Reduction Pipeline for Gemini-North's Near-infrared Integral Field Spectrometer. Astronomical Journal, 158(4), [153]. https://doi.org/10.3847/1538-3881/ab3b00, https://doi.org/10.3847/1538-3881/ab3b00

Published in:

Astronomical Journal

Document Version:

Publisher's PDF, also known as Version of record

Queen's University Belfast - Research Portal:

Link to publication record in Queen's University Belfast Research Portal

Publisher rights

(c) 2019. The American Astronomical Society. All rights reserved. This work is made available online in accordance with the publisher's policies. Please refer to any applicable terms of use of the publisher.

\section{General rights}

Copyright for the publications made accessible via the Queen's University Belfast Research Portal is retained by the author(s) and / or other copyright owners and it is a condition of accessing these publications that users recognise and abide by the legal requirements associated with these rights.

Take down policy

The Research Portal is Queen's institutional repository that provides access to Queen's research output. Every effort has been made to ensure that content in the Research Portal does not infringe any person's rights, or applicable UK laws. If you discover content in the Research Portal that you believe breaches copyright or violates any law, please contact openaccess@qub.ac.uk. 


\title{
A Data Reduction Pipeline for Gemini-North's Near-infrared Integral Field Spectrometer
}

\author{
Marie Lemoine-Busserolle ${ }^{1}$ (D), Nathaniel Comeau ${ }^{1}$, Collin Kielty ${ }^{1,2}$ (D), Kerry Klemmer ${ }^{1}$, and Megan E. Schwamb ${ }^{1}$ (D) \\ ${ }^{1}$ Gemini Observatory, Northern Operations Center, 670 North A'ohoku Place, Hilo, HI 96720, USA; bieke.decraemer@oma.be \\ ${ }^{2}$ Department of Physics and Astronomy, University of Victoria, Victoria, BC, V8W 3P2, Canada \\ Received 2018 February 14; revised 2019 July 31; accepted 2019 August 12; published 2019 September 23
}

\begin{abstract}
We present a python package, called Nifty4Gemini, and its associated Pyraf/Python based pipeline for processing Gemini-North Near-Infrared Integral Field Spectrometer (NIFS) observations. Built on the Gemini IRAF package's capabilities, Nifty4Gemini's associated NIFS pipeline is a data reduction package which reduces NIFS raw data and produces a flux and wavelength calibrate science cube with the full signal-to-noise ratio, ready for science analysis. It utilizes tasks from the Gemini IRAF package, PyRAF, and packages from the Gemini AstroConda environment. Niffy4Gemini is a configuration-based pipeline framework written in python which is easily extensible to integrate additional pipelines and user-defined scripts. Nifty4Gemini is open source and available for download at https://github. $\mathrm{com} / \mathrm{mrlb} 05 /$ Nifty4Gemini with its documentation available at https://nifty4gemini.readthedocs.io/en/latest/. A permanent version of the software described in this paper is archived at https://zenodo.org/record/1000413.
\end{abstract}

Key words: instrumentation: spectrographs - methods: data analysis - methods: observational - techniques: imaging spectroscopy

\section{Introduction}

Gemini's Near-Infrared Integral Field Spectrometer (NIFS; McGregor et al. 2003) is a facility near-infrared spectrograph which supports seeing-limited, natural guide-star adaptive optics (AOs) and laser AOs near-infrared spatially resolved spectroscopy when used with the ALTtitude conjugate Adaptive optics for the InfraRed (ALTAIR; Richardson et al. 1998), the AOs system on Gemini North (Christou et al. 2010). NIFS is currently available for use on the Gemini Observatory's 8.1-m Frederick C. Gillett Gemini North Telescope on Maunakea. The NIFS detector is a Rockwell HAWAII-2RG (H2RG) device with $2048 \times 2048$ $18 \mu \mathrm{m}$ pixels. The outer four pixels on each side are not illuminated, and so act as reference pixels. This leaves an active area of $2040 \times 2040$ pixels. The HAWAII-2RG detector is sensitive to light out to $2.6 \mu \mathrm{m}$, and uses a $\mathrm{HgCdTe}$ detector layer. It uses four output amplifiers that simultaneously read out $512 \times 2048$ pixels in around 5 s. A recorded NIFS frame is actually the difference in signal between two readouts of the detector: one at the start of the integration and one at the end. For this reason, the minimum permitted exposure time is determined by the readout time. At the heart of NIFS is a reflective integralfield unit (IFU) which divides its $\sim 3$ ". $0 \times 3$ " 0 field of view (FOV) on the sky into 29 slitlets each 0 !' 105 wide and 3!" 0 long. Each slitlet is sampled by 69 detector pixels running along the length of the slice. Spectra are obtained simultaneously for each 0!"043 pixel along each slitlet. This results in rectangular spatial pixels (or spaxels) of dimension 0 !" $103 \times 0$." 043 across and along the slice, respectively. Four reflection gratings are used with a fixed focal length camera to obtain spectra with two-pixel resolving powers of $R \sim 5300$ in any one of the $Z, J, H$, or $K$ bands.

In this paper, we describe Nifty4Gemini, a python package, which includes a data processing pipeline for NIFS observations. The NIFS pipeline was developed to reduce NIFS raw data and produce a final flux and wavelength calibrated science data cube with the full signal-to-noise needed for science analysis. It is based on pre-existing Image Reduction and Analysis Facility (IRAF) ${ }^{3}$

\footnotetext{
IRAF is distributed by the National Optical Astronomy Observatory, which is operated by the Association of Universities for Research in Astronomy (AURA) under a cooperative agreement with the National Science Foundation.
}

routines that are part of the Gemini IRAF Package, ${ }^{4}$ which is installed by AstroConda, ${ }^{5}$ and PyRAF. ${ }^{6}$ The raw data from the Gemini facility instruments are stored as Multi-Extension Flexible (MEF) Image Transport System files (FITS; Pence et al. 2010). Therefore, all the tasks in the Gemini IRAF package, intended for processing data from the Gemini facility instruments, are capable of handling MEF files. IRAF, PyRAF, and the Gemini IRAF Package have been widely used in the astronomy community and therefore have been extensively tested and are supported by Gemini Observatory. However those routines are not enough to create a pipeline, therefore we used them as a library of computer code for our NIFS pipeline, instead of rewriting the function themselves that already exist in IRAF and the Gemini IRAF Package. Nifty4Gemini and its NIFS pipeline are introduced as a software pipeline written in the python programming language, which is designed so it is easy to run and repeat the data reduction process. It is also easily extensible and straightforward to install.

In Section 2, we give an overview of the structure of Nifty4Gemini and the NIFS Pipeline. In Section 3, we describe the NIFS raw data, discuss the various steps of the data reduction that the NIFS pipeline performs as in the V1.0.1 release, and we describe the tutorials and example available in the online documentation of with Nifty4Gemini. Finally, conclusions and prospects for future development of Nifty4Gemini are presented in Section 4.

\section{General Nifty4Gemini Overview}

Our NIFS pipeline software is run via Nifty4Gemini, a python configuration-based framework which gathers input from the users, generate configuration files and control the workflow of the data reduction performed by the pipeline. The first release of Nifty4Gemini contains our NIFS pipeline and its multiple steps to carry out a data reduction (which by default

\footnotetext{
4 http://www.gemini.edu/sciops/data-and-results/processing-software/ releases

5 https://astroconda.readthedocs.io/en/latest/

6 PyRAF is a product of the Space Telescope Science Institute, which is operated by AURA for NASA.
} 
are automated). In the Nifty4Gemini framework we defined a "step" as a major part of the data reduction and each step has its self-contained Python script. Therefore each step can also be run on its own. Each step is composed of various tasks. In our framework a task is an individual PyRAF or pure Python function that does much of the data processing of a Pipeline. Each task has input data, output data, input parameters and optional return values. Nifty4Gemini uses a configuration file reader and writer named ConfigObj (version 4.7.2) ${ }^{7}$ to create and load a configuration file, called config.cfg. All data reduction parameters used by the NIFS pipeline are saved in the config.cfg file, which is stored and can be shared, making it easy to exactly reproduce a data reduction workflow. Nifty4Gemini is open source ${ }^{8}$ and the git repository includes the source code with detailed installation instructions, a quick-start guide, and a link to the full documentation, which is hosted on ReadTheDocs. ${ }^{9}$ A permanent version of the software is archived and available at https://zenodo.org/ record/1000413.

\section{NIFS Data Reduction Pipeline}

We present here a typical NIFS data set and describe the major steps of our NIFS pipeline to reduce and calibrate such observations (Figure 1).

\subsection{Required Data Set}

Most NIFS observations use a standard setup with science and associated sky frames as well as a basic set of frames for calibration measurements, which we refer as baseline calibrations. The baseline calibration set usually includes: (1) spectral flat-field frames (lamps-on and lamps-off frames), which are required for each grating setting in order to correct pixel-to-pixel gain variations in the spectrograph detector and the wavelengthdependent throughput of the spectrograph (including the wavelength-dependent response of the optics, filter, grating, and detector); (2) distortion calibration frames obtained using a coarse Ronchi grating, which are used to calibrate the spatial dimension of the IFU field; the Ronchi flat frame is a dispersed flat-field image with a slit-mask in the IFU field so that the illumination on the IFU is in a pattern of $\sim 10$ different slitlets that are stacked in the $y$-dimension.; (3) Wavelength calibration frames, based on exposures of arc lamps, which are also obtained each time the grating turret in NIFS is moved; (4) dark frames obtained in order to subtract the dark current pattern from the arc frame; and (5) four exposures on-source of a smooth spectrum star are generally obtained together with two sky frames of the same exposure time. Those frames can be used to correct telluric absorption lines and also used for flux calibration if that telluric star's broadband near-infrared magnitudes are known. It is worth noting that each science data set and Telluric data set also comes with associated acquisition images, used to center the science target in the NIFS FOV. All NIFS raw MEF data files have two unnamed extensions. Most of the header information is written to the primary header unit (PHU), extension [0]. The data read from the detector array is in extension [1].

\footnotetext{
http://www.voidspace.org.uk/python/configobj.htm

8 https://github.com/mrlb05/Nifty4Gemini

9 https://nifty4gemini.readthedocs.io/en/latest/
}

\subsection{Step 1: Getting and Sorting the NIFS Raw Data}

The raw NIFS observations can either be located in a local directory or the pipeline can download them from the Gemini Observatory Archive ${ }^{10}$ (if the data are public) by providing a program ID (e.g., GN-2017B-Q-1). In the latter case, the raw data will be automatically downloaded, verified (by ensuring that a minimum of data was found for science target, calibration, and telluric star), and decompressed. Nifty4Gemini uses some components of NDMApper ${ }^{11}$ for this purpose. The first step of the data reduction sorts the raw NIFS data in appropriate directories according to their types, date obtained, and grating configurations. During this step, using the information present in headers of each MEF file, the pipeline creates a new directory structure, text files with list of files names by types (e.g., calibrations, science target, telluric star, science target acquisition), and will write the paths to the newly created directories in the configuration file config.cfg. It is important to note that for NIFS there is no difference in the headers between lamps-on flats (we refer to as flats) and lampsoff flats (we refer to as flatdark). For all flats, the pipeline computes an average count and if the median is greater than 2000 , the image is identified as a flat and if it is less it is identified as a flatdark. An example of raw calibration and science frames are shown in Figure 2. All data reduction figures use $K$-band observations of Titan (program GN-2014A-Q-85; obtained in 2014 April/May) as an example.

The pipeline also at this stage associates science frames with telluric star frames that are closest in time (less than $1.5 \mathrm{hr}$ ) and stores this information in a text file to be used later in the data reduction process. This step needs to be run only once unless additional raw data are added. All tasks in this first step are written entirely in python.

\subsection{Step 2: Reducing the NIFS Raw Calibration Data}

The second step is to reduce the NIFS baseline calibration observations. This sequence is performed on the calibration data located in each calibration directory. Step 2 is composed of four tasks, where each of them are a python wrapper around Gemini IRAF tasks. During the first task, iraf.nfprepare from the Gemini IRAF package is used to determine the image slicer offsets and to calculate variance and data quality frames. Headers values in the observation determine the observation mode and so identify a particular mask definition file (MDF). The MDF describes the illumination pattern on the detector of the image slicer and nfprepare uses it to compute any spatial shift of the IFU maps on the detector from the standard MDFs for each NIFS observation. We refer to the output as the shift frame. In addition, using the information in the MDF, three extensions are extracted from the original raw data: the data (SCI), variance (VAR), and data quality (DQ). The generation of the DQ plane is important in order to identify and fix hot and bad pixels on the NIFS detector in subsequent steps in the data reduction process. Pixels with analogue-to-digital unit (ADU) values that exceed the linear limit of the detector $(\sim 35,000$ ADU), or pixels which are saturated $(\sim 48,000$ ADU), are flagged within the DQ frame at this step in the reduction process. In addition, the use of nfprepare and iraf.nffixbad will minimize the effect of bright cosmic-ray strikes by linear

\footnotetext{
${ }^{10}$ https://archive.gemini.edu/searchform

11 https://github.com/jehturner/ndmapper
} 


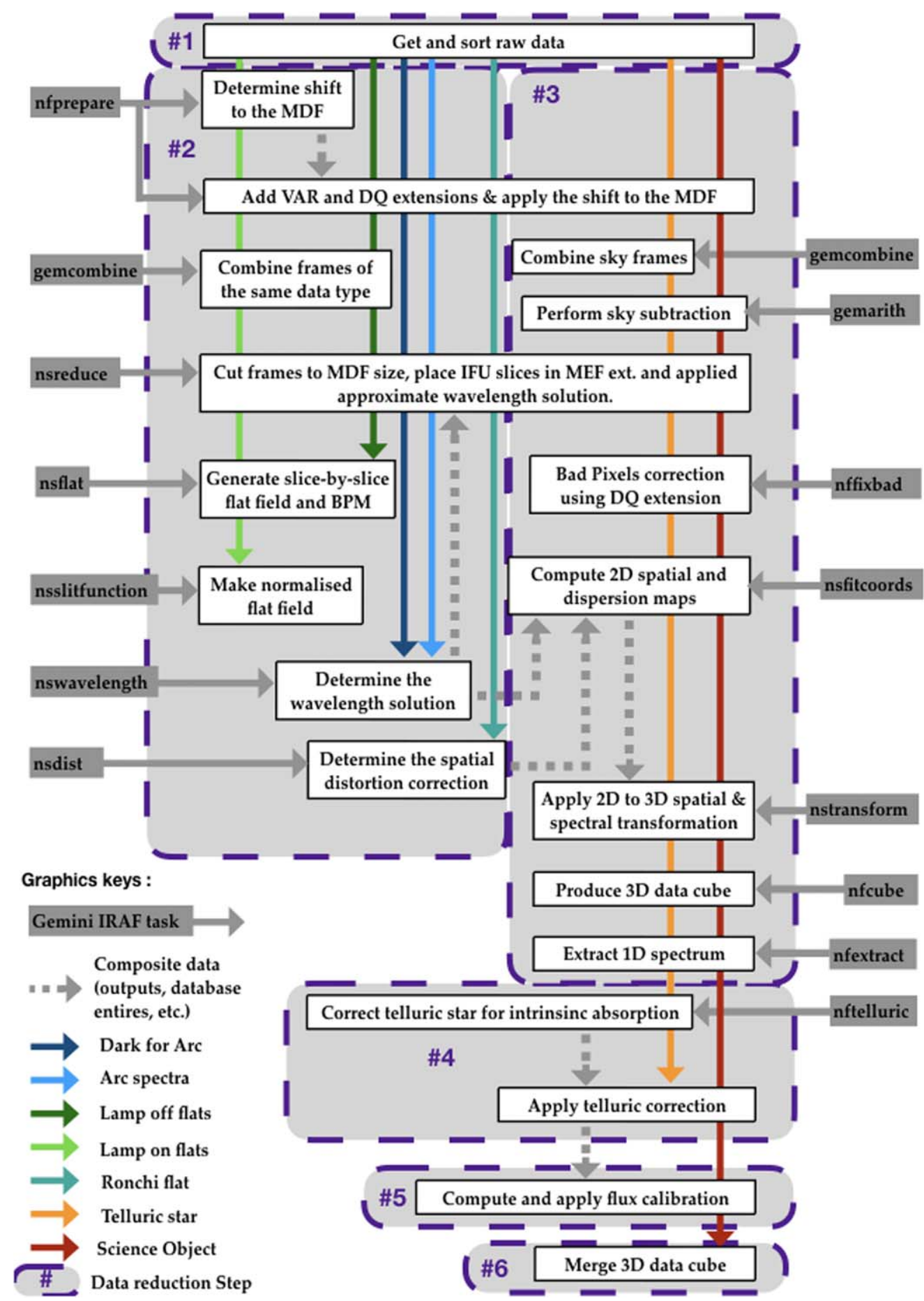

Figure 1. NIFS pipeline's workflow which includes the Gemini IRAF tasks used during the different steps. There is no Gemini IRAF task used for the last step of the data reduction.

interpolation using the nearest good pixels, following the information in the VAR and DQ planes. Cosmic-ray effects are also diminished with iraf.gemcombine via median combination of multiple frames of the same data type, and iraf.nifcube will further suppress their effect when merging reduced data cubes from multiple science acquisitions (see Section 3.7).

In the second task, all lamps-on and lamps-off flats are run through iraf.nfprepare with the shift frame used as a reference to apply the MDF shift. The variance and quality extensions are also generated. All lamps-on flats are combined into a master lamps-on flat using iraf.gemcombine and all lamps-off flats are combined in a similar fashion. iraf.nsreduce is then used on the two combined lamps-on and lamps-off flats frames (1) to cut the frames to the size specified in the MDF, (2) to place each IFU slice in separate MEF extensions, and (3) to apply an approximate wavelength calibration. ${ }^{12}$ The outputs are then run through iraf.nsflat to generate a normalized slice-by-slice flat-field frame and a slice-by-slice bad pixel mask (BPM)

\footnotetext{
12 This approximate wavelength solution is only needed within iraf.nsreduce and is not reflected in the science frames; proper wavelength calibration is done at a later stage.
} 


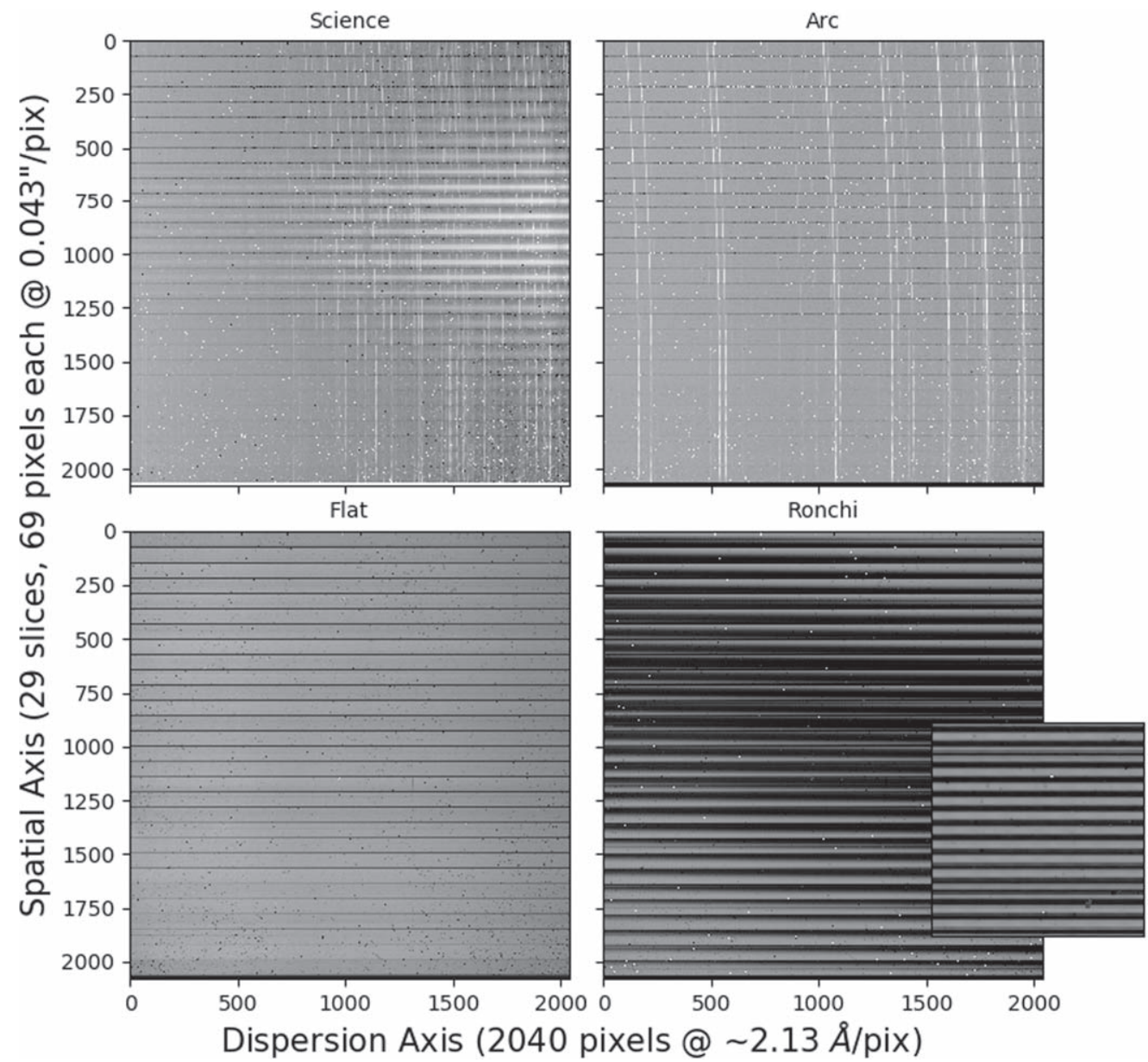

Figure 2. Example of raw NIFS $K$-band science and calibration (lamp-on flat, lamp-on dark, and Ronchi) frames in the detector plane. Easily seen in the flat frame (bottom left), the dark horizontal lines outline the edges of each IFU slice. A zoom in region of the Ronchi frame is shown to display the Ronchi slit-mask (bottom right).

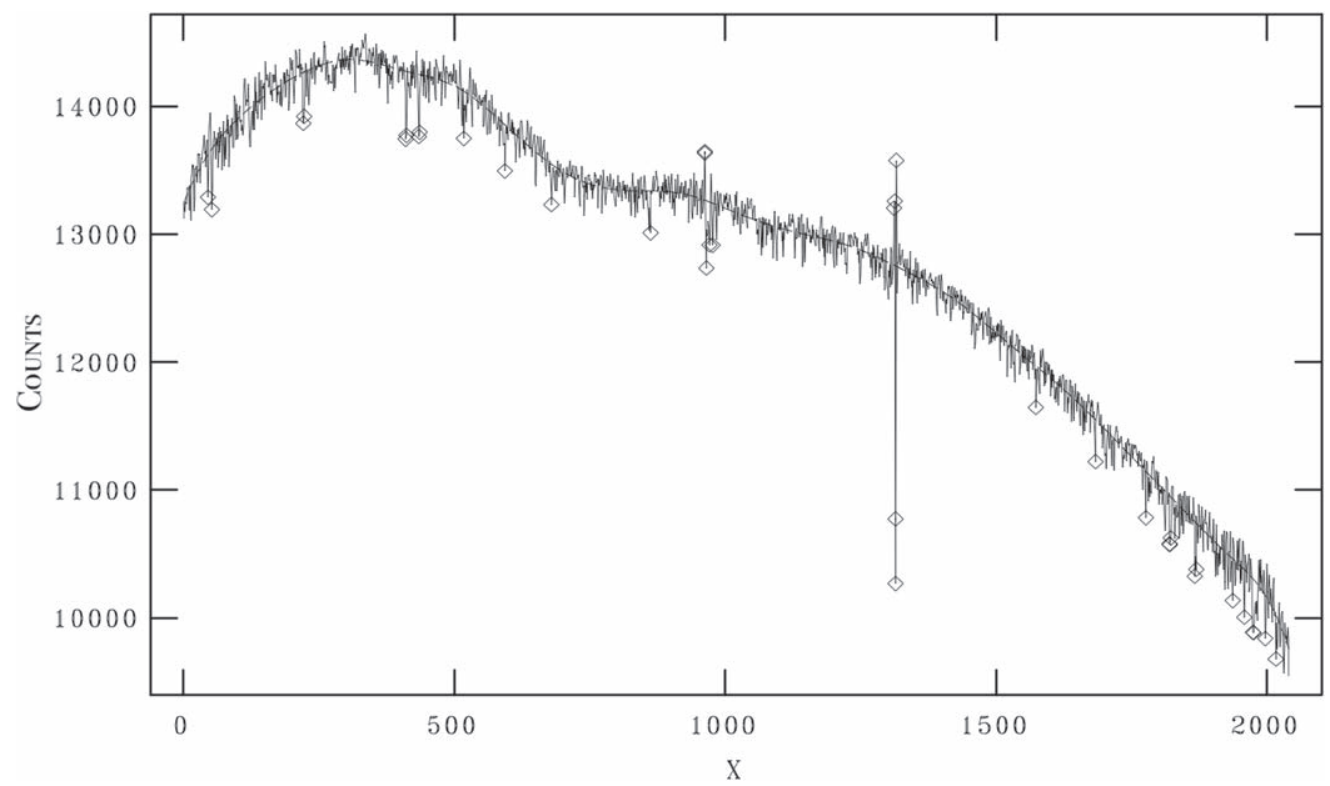

Figure 3. Example of fitting a $K$-band lamps-on flat spectrum using iraf.nsflat. Pixels rejected via thresholding are identified by open diamonds and an order 20 cubic spline is fit to normalize the flat field.

frame via thresholding (see Figure 3). In order to ensure that the number of bad pixels caught is approximately constant and correct and does not depend of the wavelength, the pipeline has different default lower rejection threshold values for each grating in the creation of the BPM. The process we used to select the optimal rejection threshold value for each band was as follows: 


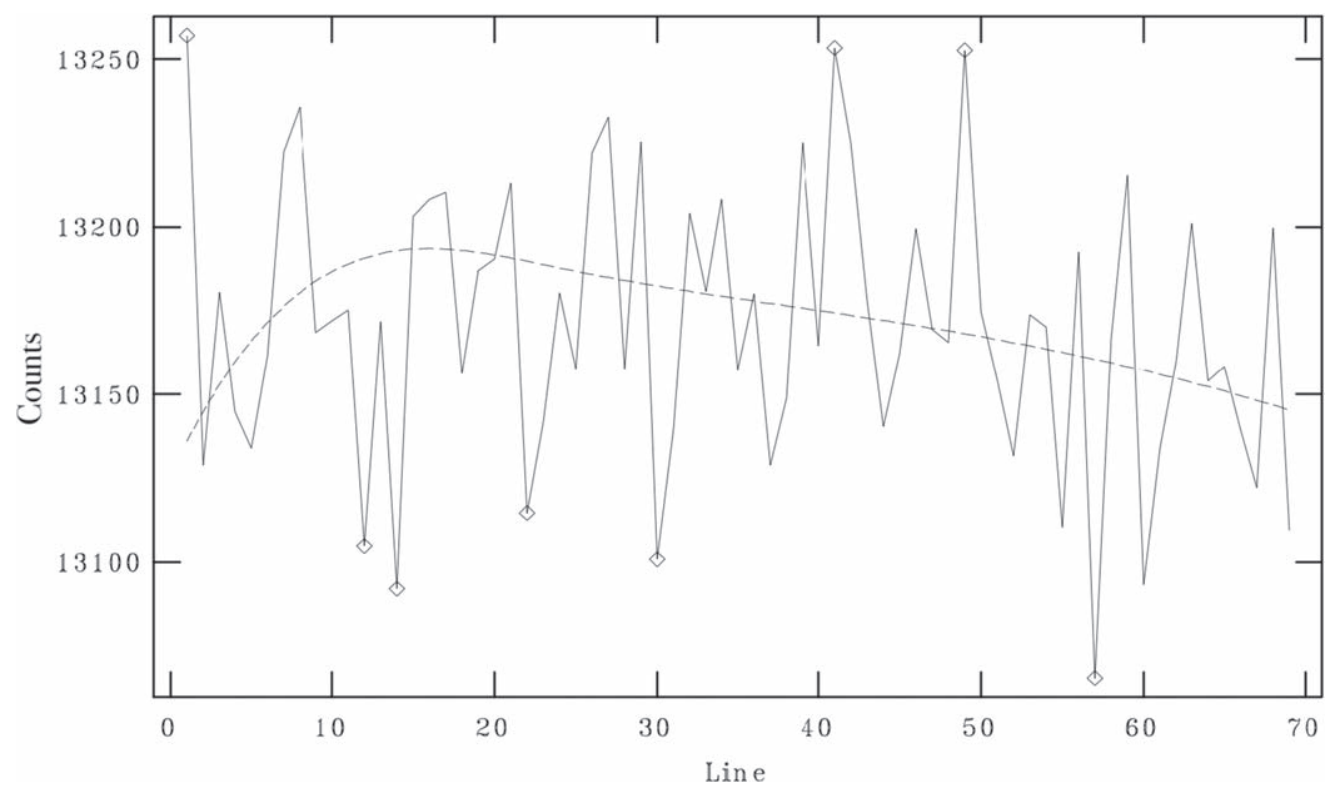

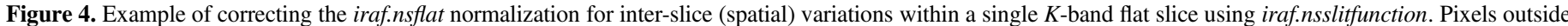
$2 \sigma$ of the median are rejected and identified by open diamonds. An order of 3 cubic spline is fit to further normalize the flat field on a slice-by-slice level.

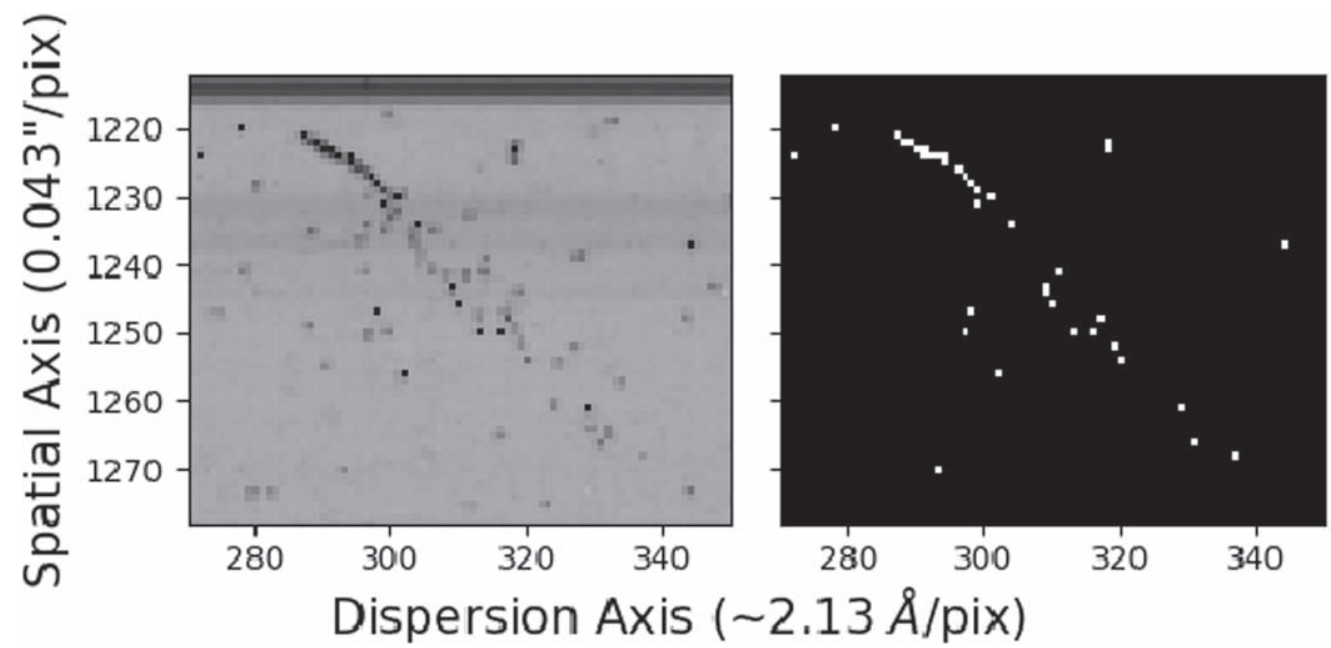

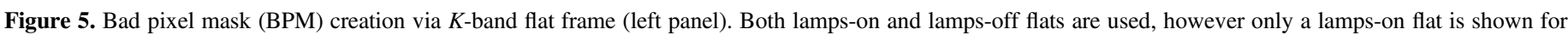

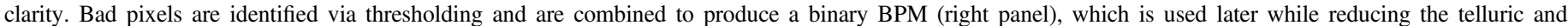
science data.

(1) a set of DQ frames was produced for a given grating, utilizing a range of rejection threshold values; (2) the number of bad pixels identified in each frame, for a given threshold, is recorded and compared to other frames processed using the same threshold; (3) the threshold that rejects the most consistent number of pixels between frames is chosen for that grating (typical rejection count differences between frames are on the order of 10 pixels); (4) steps 1-3 are repeated for all gratings; and (5) the number of rejected pixels for each grating is compared and the thresholds are iteratively adjusted until the number of pixels rejected between gratings is comparable to the number of pixels rejected between frames of a particular grating (on the order of 10 pixel differences). Lower rejection thresholds, in terms of a fraction of the median value in the frame, are 0.07 for the $Z$ and $J$ gratings and 0.05 for the $H$ and $K$ gratings.

The final flat-field frame and BPM are created with iraf. nsslitfunction (Figure 4) by correcting the normalization done by iraf.nsflat for slice-to-slice variations. The output from this task is used as the flat-field image and BPM (Figure 5) for further reduction.

In the third task, similarly as for the lamps-on and lamps-off flats, the arc lamp frame (called arc frame) and associated dark frame (called arc dark frame) are run through iraf.nfprepare and iraf.gemcombine (if there are more than one arc lamp and arc dark frame) after those frames are processed by iraf. nsreduce with the flat-field being applied. The wavelength solution for each slice is obtained by running iraf.nswavelength, which uses the core IRAF tasks identify/reidentify.

iraf.nswavelength calls custom high-resolution line lists designed for the Gemini calibration unit (GCAL), which are suitable for the spectral resolution of NIFS. These line lists have been vetted by eye to remove weak/blended lines that obfuscate automatic line identification. The line list which best covers the wavelength regime of the grating in use is loaded. 


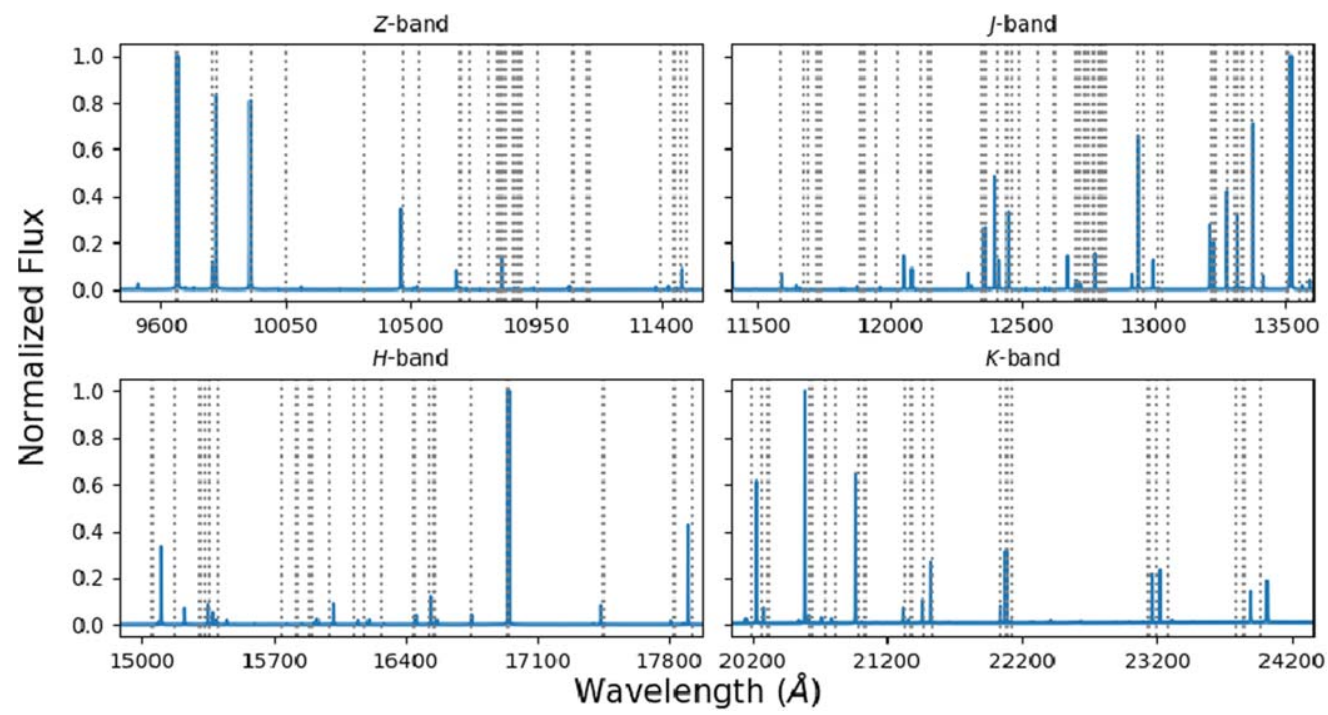

Figure 6. Example of an 1D arc spectra (blue) and known arc line positions (dashed gray) for each of the four NIFS gratings. The arc spectra shown have been processed with iraf.nsreduce, giving only an approximate linear wavelength solution. Arcs are used by iraf.nswavelength to compute the wavelength solution (see Figure 7).

Table 1

Nifty4Gemini Wavelength Calibration Summary

\begin{tabular}{lccccr}
\hline \hline Grating & $\begin{array}{c}\text { Band Pass } \\
(\mu \mathrm{m})\end{array}$ & Calibration Lines & $\begin{array}{c}\text { Line List Coverage } \\
(\mu \mathrm{m})\end{array}$ & $\begin{array}{c}\text { Peak Threshold } \\
\text { ADU }\end{array}$ & $\begin{array}{c}N_{\text {lines }} \\
\left(\mathrm{km} \mathrm{s}^{-1}\right)\end{array}$ \\
\hline$Z$ & $0.94-1.15$ & $\mathrm{Ar}, \mathrm{Xe}$ & $0.94-1.14$ & 100 & $11.7 \pm 2.9^{\mathrm{a}}$ \\
$J$ & $1.15-1.33$ & $\mathrm{Ar}$ & $1.16-1.32$ & 100 & 33 \\
$H$ & $1.49-1.80$ & $\mathrm{Ar}$ & $1.51-1.79$ & 100 & 40 \\
$K$ & $1.99-2.40$ & $\mathrm{Ar}$ & $2.02-2.39$ & 50 & 24 \\
& & & & $2.8 \pm 1.4$ \\
\hline
\end{tabular}

Note. Wavelength coverage, line list information, and typical rms error in the wavelength solution for Nifty4Gemini.

${ }^{\mathrm{a}}$ When run automatically (not default); can be minimized with interactive line identification.

Figure 6 shows typical arc spectra for each of the four gratings with the line list coverage overlaid. A line list summary and typical rms errors on the wavelength solution for each grating are given in Table 1. Thresholding parameters are set in iraf. nswavelength to optimize the wavelength solution for the NIFS spectral resolution ( width $=2$, cradius $=8$; the maximum distance, in pixels, between a line position and the initial estimate) as well as to optimize the solution for each grating based on the intensity of the arc lines (see Table 1).

The pipeline has predefined input to determine the wavelength solution automatically for the default configurations of the $J, H$, and $K$ gratings. Though the line list used for the $Z$ grating covers the full band (see Figure 6 and Table 1), iraf.nswavelength often fails when run automatically on $Z$-band data. For the $Z$ grating and nonstandard wavelength configurations (i.e., the $K$-long or $K$-short gratings, or nonstandard choices in central wavelength), iraf.nswavelength will run interactively and will wait for user input as required. iraf. nswavelength does not directly calibrate the data but outputs a series of files in a "database/" directory containing the wavelength solutions to be used later by iraf.nstransform. An overview of the iraf.nswavelength process for an example $K$ band arc is shown in Figure 7.

In the fourth and last task of the NIFS baseline calibration observations reduction, the Ronchi flat frames are respectively being run through iraf.nfprepare, combined with iraf.gemcombine, and processed with iraf.nsreduce, which also applies the flat field.
Finally, iraf.nfsdist uses the information in the Ronchi flat calibration image to calibrate the spatial dimension of the NIFS IFU field on the detector. In fact, the measure of the spatial mapping for the images slices with iraf.nfsdist is required for accurate alignment of the slices (see Figure 8). Proper alignment of the slitlets across each slice is used for spatial rectification of the NIFS on-sky data. The output of iraf.nfsdist is a spatially referenced Ronchi flat with its associated alignment files located in the "database/" directory. The spatial solution determined by iraf.nfsdist will be linked to the science and telluric star data by iraf.nsfitcoords in a later step.

In summary, the outputs of a full NIFS baseline calibration observation reduction are (1) an MDF shift frame, (2) a reduced flat-field frame, (3) a BPM frame, (4) a wavelength referenced arc frame, and (5) a spatial calibration Ronchi flat frame (with an associated database directory, for each of (4) and (5), containing information on the wavelength solution and the spatial distortion correction). All of these products are stored in the relevant science target and telluric star observation directories. In the case of standard wavelength configurations observations, the full NIFS baseline calibration observation reduction is run automatically.

\subsection{Step 3: Common Steps of the Science Target and Telluric Star Reduction}

This stage of the NIFS pipeline is common to both the science target data and the telluric star observations and is done 

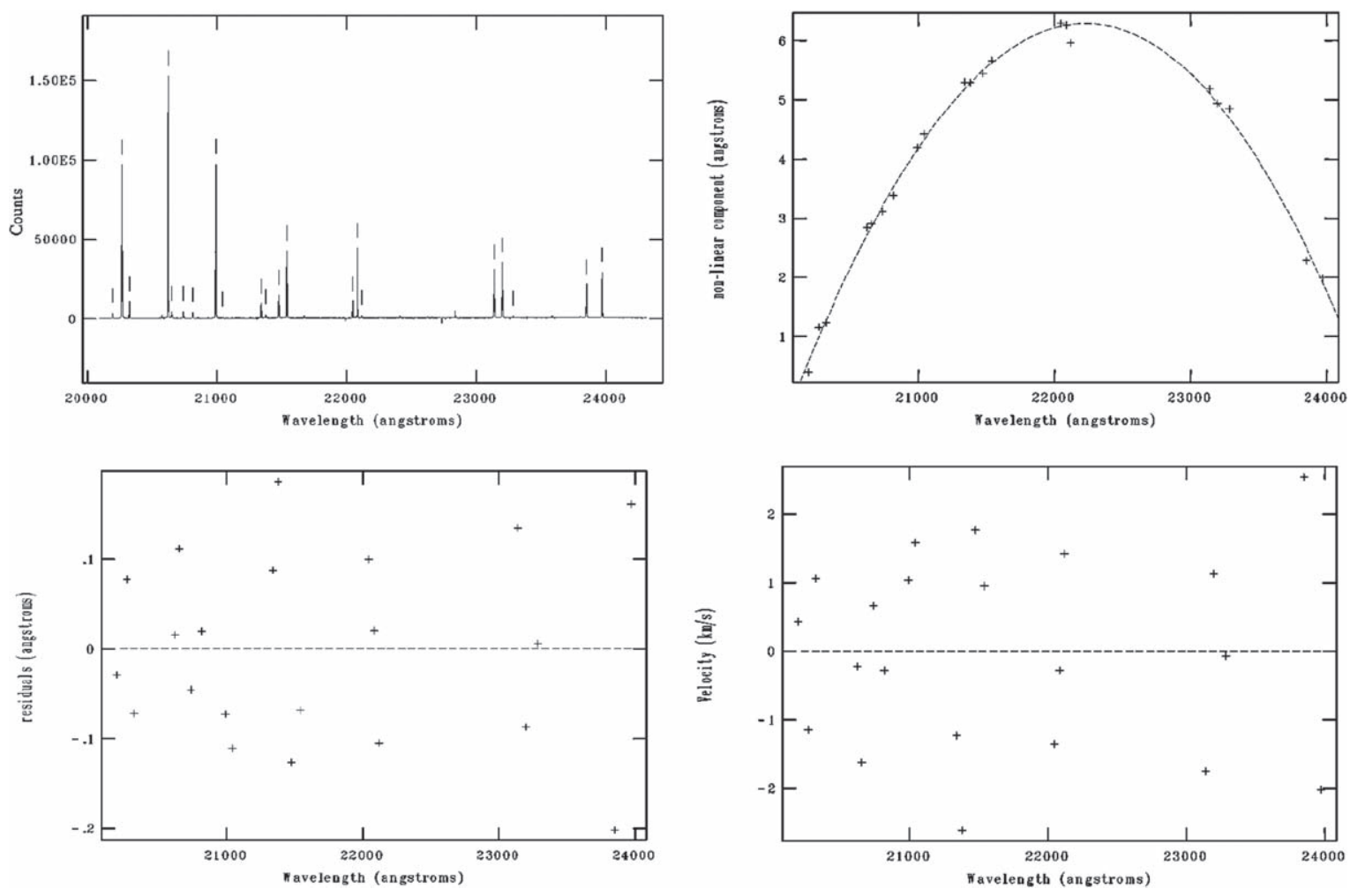

Figure 7. Example output for iraf.nswavelength when run on a $K$-band arc. Emission peaks in the arc spectrum are identified and matched to known lines in the line list (top left panel). Each identified line is shifted to the rest-frame wavelength and a fourth order chebyshev polynomial is fit to the shifts producing a nonlinear wavelength calibration across the full frame (top right panel). The wavelength solution is applied to the arc and the positions of the identified line are remeasured. This process is repeated until the line-by-line residuals converge (bottom left and right panels; residuals in pixels and $\mathrm{km} \mathrm{s}^{-1}$, respectively).
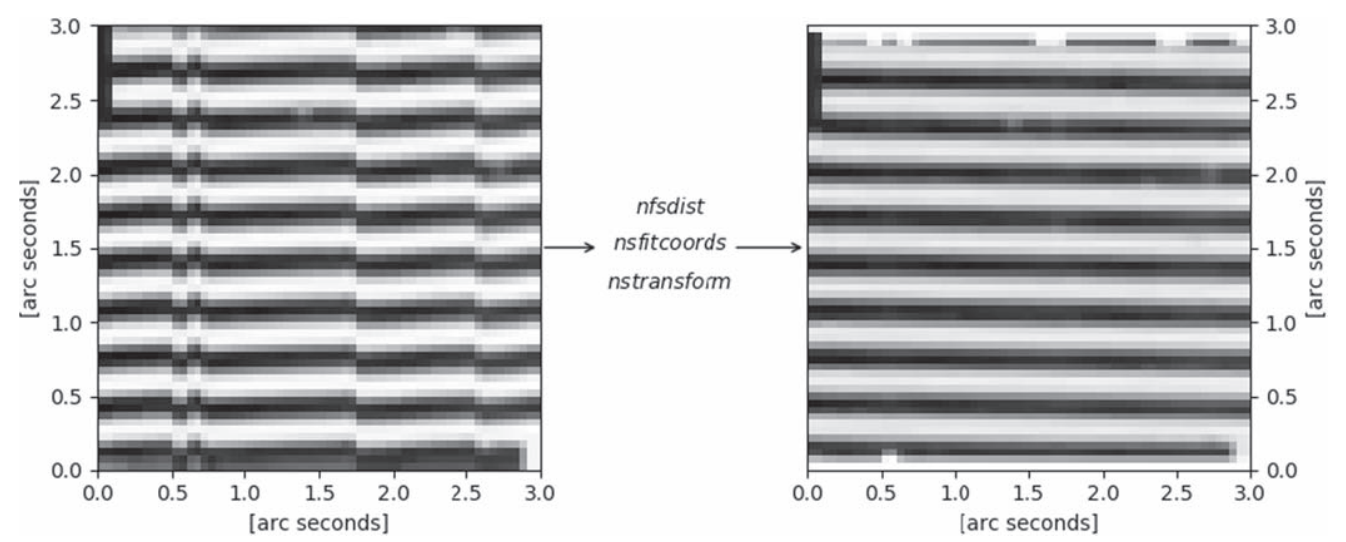

Figure 8. Example of Ronchi frames reconstructed into the sky/telescope plane to demonstrate spatial rectification. The raw frame (left) is processed with iraf.nfsdist, iraf.nsfitcoords, and iraf.nstransform (see Section 3.4) to produce a Ronchi flat that is specially rectified in the sky plane (right).

on each set of files located in each science target and telluric star data directories. Step 3 contains eight tasks which are python wrappers around tasks of the Gemini IRAF package.

In this step's first task, all science targets and telluric stars data are processed with iraf.nfprepare with the shift image as a reference to apply the MDF shift and add the VAR and DQ extensions. In the second task, sky subtraction is performed. In case of the telluric data, since exposure times are usually short, the sky frames are median-combined with iraf.gemcombine. The output combined sky frame is then subtracted from each telluric frame with iraf.gemarith. In the case of the science data only the closest sky frame in time is subtracted from each science frame with iraf.gemarith. In the third task, iraf.nsreduce is used to (1) to cut the frames to the size specified in the MDF, (2) to place each IFU slices in separate MEF extensions, (3) to apply the flat field, and (4) to apply an approximate wavelength calibrations. And iraf.nffixbad uses the information in the DQ extension, which flagged bad pixels, to correct them by linear interpolation to the nearest pixel not identified as bad along the spatial axis. In the next task, the pipeline computes slice-by-slice 2D dispersion and distortion maps with iraf.nsfitcoords by fitting a third order chebyshev polynomial to the spatial rectification traces from iraf. $n f s d i s t$ and to the wavelength solution deduced with iraf. nswavelength.

Then iraf.nstransform applies the spatial and spectral transformation determined previously by iraf.nsfitcoords to produce rectified 3D data on a uniformly sampled wavelength scale. 

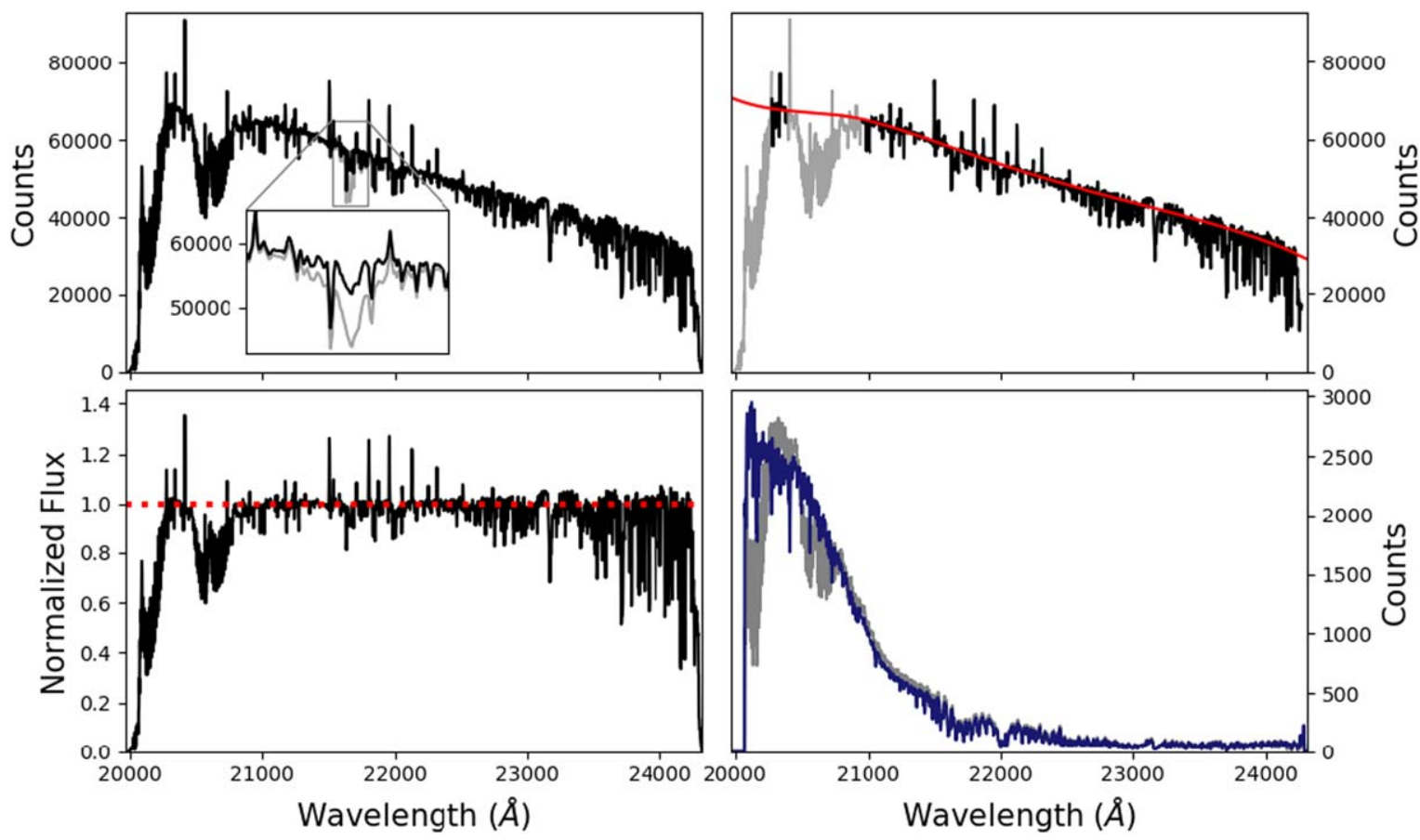

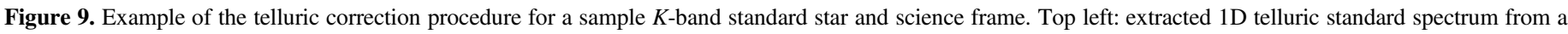

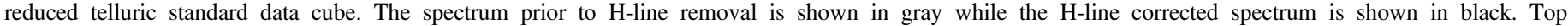

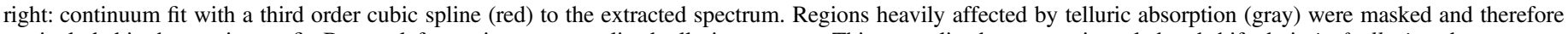

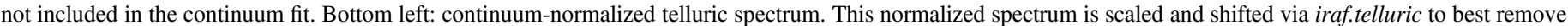

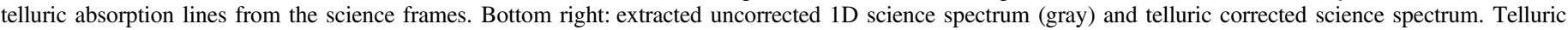
corrections are performed on the individual science exposures before merging the cubes.

The fifth task uses iraf.nifcube to produce a 3D data cube FITS file for each science target and telluric stars frame. iraf. nifcube takes input from data output by iraf.nstransform and by iraf.nsfitcoords and converts the 2D data frames into data cubes that have coordinates of $x, y$, and $\lambda$ and a square pixel size of 0 ."05 on the sky using a 3D Drizzle algorithm. Nifty4Gemini calls iraf.nifcube with the default input parameters set by the Gemini IRAF Package. A full description of iraf.nifcube's functionality, input parameters, and algorithm is given in Appendix A.1. A 1D spectrum is extracted from each data cube with iraf.nfextract using a circular aperture (the radius is an input parameter in the configuration file), and the pipeline combines all 1D spectra obtained for each observation number for each science target and telluric star, respectively. This functionality was added to the pipeline due to a need for a quick and easy way to extract from the data cube 1D spectrum for a source located at the center of the NIFS field for some specific type of science. Users should be aware that these 1D spectra are not useful in other cases like (1) extended sources with unknown shape and/or (2) while trying to discover faint emission in the data cubes. In the first case, active contours or other image segmentation methods from computer vision may be useful. In the second problem, see, e.g., the DUCHAMP 3D source finder developed for SKA-precursors by Whiting (2012).

\subsection{Step 4: Derive and Apply Telluric Absorption Correction to Science 3D Data Cubes}

The first task of the telluric correction is to determine the telluric star spectral type, temperature, the Two Micron All Sky Survey (2MASS; Skrutskie et al. 2006) magnitude by querying Set of Identifications, Measurements, and Bibliography for
Astronomical Data (SIMBAD; Wenger et al. 2000) and the observation exposure time from the fits files headers. All these values are then written in a text file and will be used in the next step to flux calibrate the telluric absorption corrected science data cubes. The pipeline assumes that a bright, early A-type star was observed to cancel out telluric absorption. iraf.telluric is used to remove intrinsic absorption lines from the standard star spectrum by shifting and scaling a $R \sim 5000$ spectrum of Vega as a model. The shift and scale values are written out to a text file. While it is recommended to use an early A-type star as the telluric standard, if an alternate type of star is chosen, users may specify a custom reference spectrum to be used by iraf. telluric. Similarly, the pipeline also provides the option to not remove any intrinsic lines from the telluric star and skip this task. In that case, the assumption is that either the science will not be affected by those intrinsic lines or the user will provide a telluric star spectrum already corrected if telluric correction will be performed. After intrinsic absorption is removed, a normalized telluric spectrum is produced by (1) fitting a third order cubic spline to regions not affected by telluric absorption in the telluric star spectrum and (2) by dividing the telluric star spectrum by this continuum fit (see Figure 9). Regions free of telluric absorption, in each grating, have been identified by eye and are incorporated into the pipeline, however we encourage users to fit these regions interactively for higher fidelity telluric correction.

In the final task, iraf.telluric is used to remove the telluric absorption from the 3D science data cube by shifting and scaling the normalized telluric spectrum and dividing it from the science cube (see Figure 9). While iraf.telluric performs an iterative fit to optimize the telluric absorption correction, this process is fundamentally limited as only a single shift and scale 


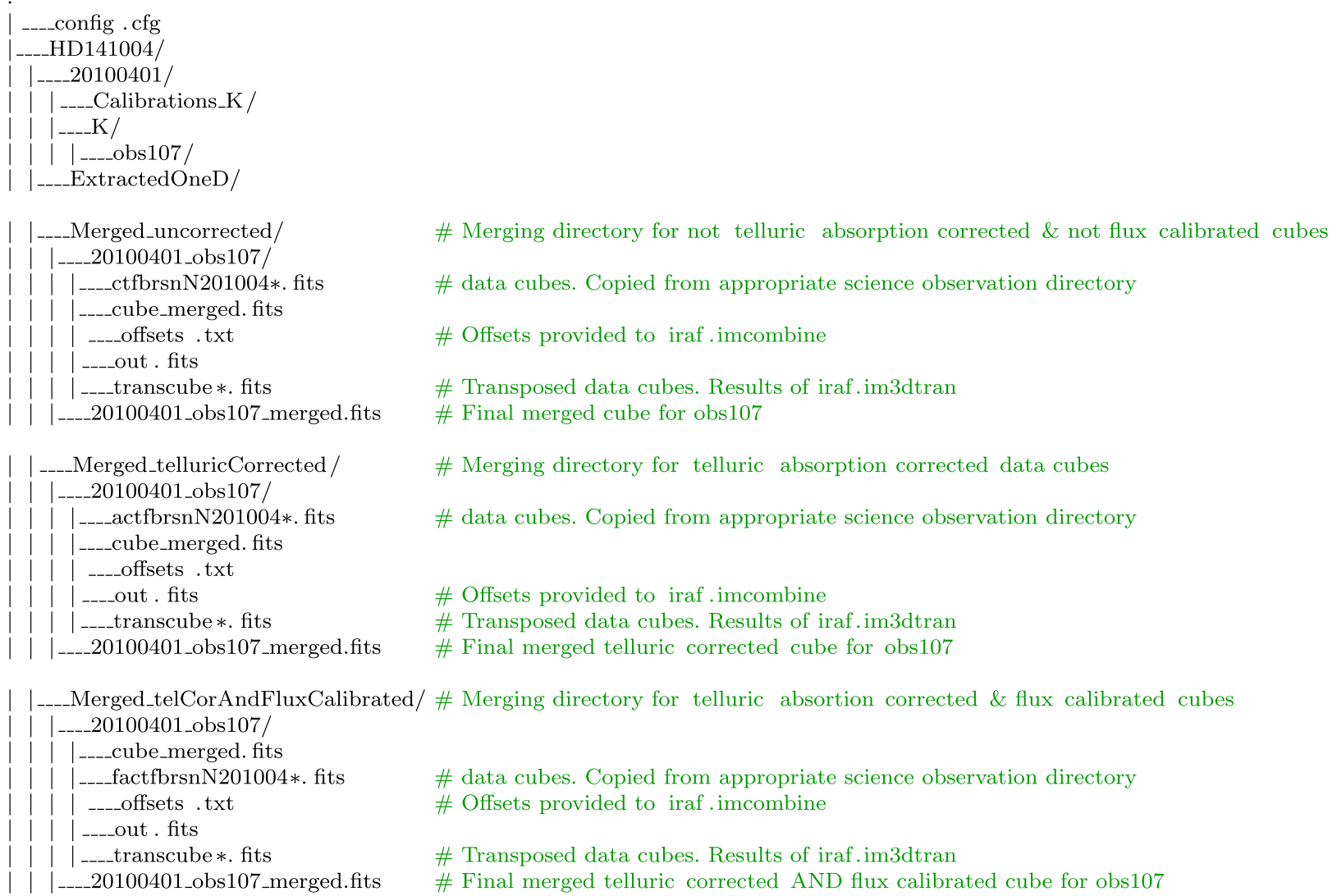

Figure 10. Example of the merging directories after running the pipeline to get merged 3D science data cubes for a single observation (number 107) taken on a single night (2010 April 4).

value is ultimately used. A proper treatment of telluric removal, by assessing each telluric line individually, is encouraged for users who require high-precision telluric correction.

\subsection{Step 5: Flux Calibrate the Telluric Absorption Corrected Science 3D Data Cubes}

Flux calibration for NIFS spectra is performed as in version 1.9 of the XDGNIRS pipeline (Mason et al. 2015). A blackbody spectrum is produced using the known temperature of the standard star and is then scaled to the standard star's 2MASS magnitude (Skrutskie et al. 2006). This scale is then applied to all science cubes. Assuming A-type stars have been used for the telluric standard, this assumption is reasonable as the nearly featureless spectra of A-type stars in the infrared closely match the continuum spectrum of a blackbody of the appropriate temperature (Pecaut \& Mamajek 2013). ${ }^{13}$ Mason et al. (2015) assessed the accuracy of this calibration by observing multiple standard stars on a single night and using one star to flux calibrate the other(s). Flux errors of 5\%-40\% were observed, with seeing variations within the 0". 3 GNIRS slit contributing the largest source of error.

\subsection{Step 6: Merge All Science 3D Data Cubes of the Same Target}

The Gemini IRAF Package does not provide any task to merge the $3 \mathrm{D}$ science cubes and this process can be challenging

\footnotetext{
${ }^{13}$ An interactive approach to the flux calibration is encouraged if the observer chooses to use a telluric standard of an alternative spectral class.
}

if there are many data cubes obtained on different nights. Thus, Nifty4Gemini's NIFS pipeline provides an efficient and flexible process to merge all science 3D data cubes of the same target into a final cube. This step is composed of six tasks, which can be used to combine the three different types of 3D science data cubes produced by the pipeline: (1) data cubes that are produced by iraf.nifcube at the end of step 3 of the data reduction process, (2) data cubes which have been corrected from telluric absorptions, and (3) data cubes which have been corrected from telluric absorptions and flux calibrated. The users can chose to do all of these tasks. It is important to note that none of these tasks changes the data cubes pixel size produced by iraf.nifcube, and the only combining operations performed on the data cube's pixels are those done by iraf. imcombine only and the choices are average, median, and sum.

In addition, the pipeline currently has three ways to combine 3D data cubes. In the first case, the pipeline uses the Gemini coordinates system for telescope offsets defined as $(p, q)$, which are in arcsec, and read the $p$ and $q$ values from the MEF file headers. The pipeline goes through all the directories which contain science 3D data cubes, and makes a list of the files names. After it copies each of the files in subdirectories under a new created top directory called Merged. The subdirectories's names are the observation IDs. For each subdirectory, the $(p, q)$ values from the first cube in the list is used as the reference. Thus, the pipeline computes the $x$ and $y$ offsets from these references values for each of the remaining cube from the list. Those offsets are converted into pixels and stored in files called offsets.txt. The references $(p, q)$ values are recorded as $(x=0$, $y=0)$ in pixels size in the offsets.txt file. The third axis, 


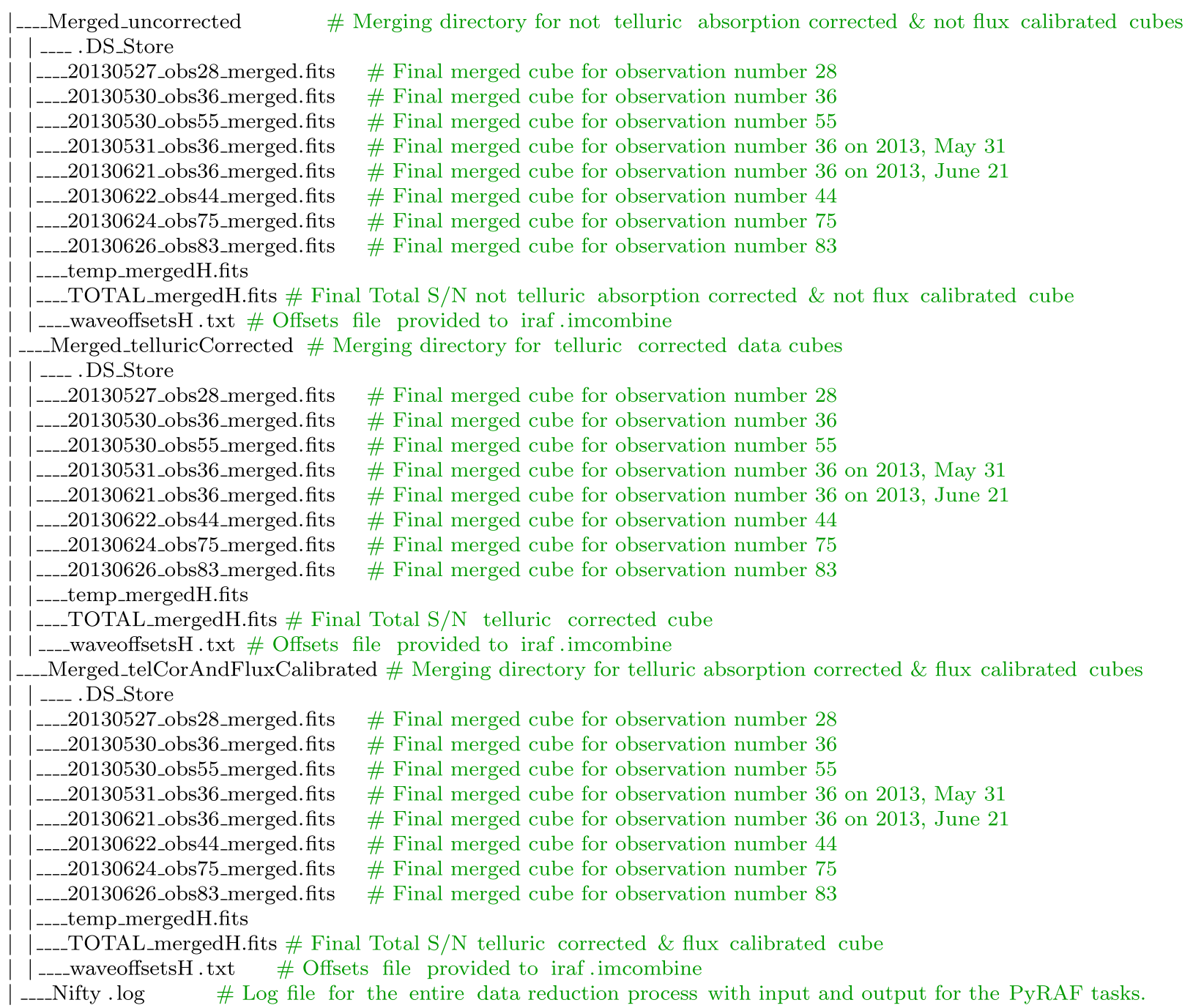

Figure 11. Example of a directory structure showing all merged 3D science cubes as well as the total $\mathrm{S} / \mathrm{N}$ merged cube for the three different types of science data cube produced; not telluric absorption corrected and not flux calibrated, only telluric absorption corrected and telluric absorption corrected and flux calibrated.

corresponding to the wavelength, is also recorded as " 0 " for all cubes. After the pipeline uses iraf.imcombine to apply the shifts in the offsets.txt to all data cubes before combining them into a single 3D data cube. However before this step the pipeline, by default, swaps the lambda and $y$-axis using iraf.im3dtran (the offsets.txt format is $(x, \lambda, y))$. We found that if this is done iraf. imcombine runs 50 times faster, otherwise it can take 25 minutes to shift and merge cubes. We have verified that cubes produced with and without using iraf.im3dtran are identical. The users can decide or not to use iraf.im3dtran. The pipeline will swap back the lambda and $y$-axis after the merging. The second way to combine the $3 \mathrm{D}$ cubes is to provide an already completed offsets.txt file. In this case, the pipeline reads in the offsets file and uses it with iraf.imcombine as described before. As the third way, the user can provide already shifted 3D data cubes. In that case, while scanning each of the directories with science 3D data cubes, if the pipeline find files names which start with "shift" it will directly combine them with iraf. imcombine. Figure 10 shows an example of the merging directories after running the pipeline to get merged 3D science data cubes for the three types of science data cubes. In this example, there is only one single observation (e.g., observation 107 ), and then the pipeline produced merged cubes for telluric absorption corrected and flux calibrated cubes, telluric absorption corrected only cubes and cubes with no telluric absorption correction and no flux calibration done.

Once a merged cube has been produced for each subdirectory, a list is created of all merged cubes for each day observations were taken with the same grating configuration. The pipeline then selects the first cube in the list as the reference $(x=0, y=0)$ and computes the $x$ and $y$ offsets from these reference values for each of the remaining cube from the list. It also computes any wavelength offsets from the other cubes and includes them in the text file containing the offsets (for example waveoffsetsK.txt). Finally, the pipeline uses iraf. imcombine to create the final 3D data cube, by merging all those cubes. This last merged data cube should have the full signal-to-noise ratio $(\mathrm{S} / \mathrm{N})$ from all science exposures of a given NIFS grating configuration for the same science target. Figure 11 shows an example of the merging directories after running the pipeline to get merged $3 \mathrm{D}$ science data cubes for each day observations were taken, and after the pipeline produced a final full $\mathrm{S} / \mathrm{N} 3 \mathrm{D}$ science cube with the same grating configuration for a single science target.

Figure 12 shows an example of the merging process for a nonsidereal target, Saturn's moon Titan. In this case, because 

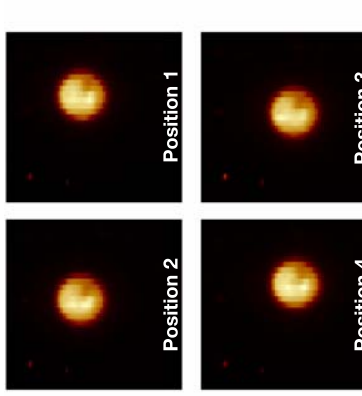

.
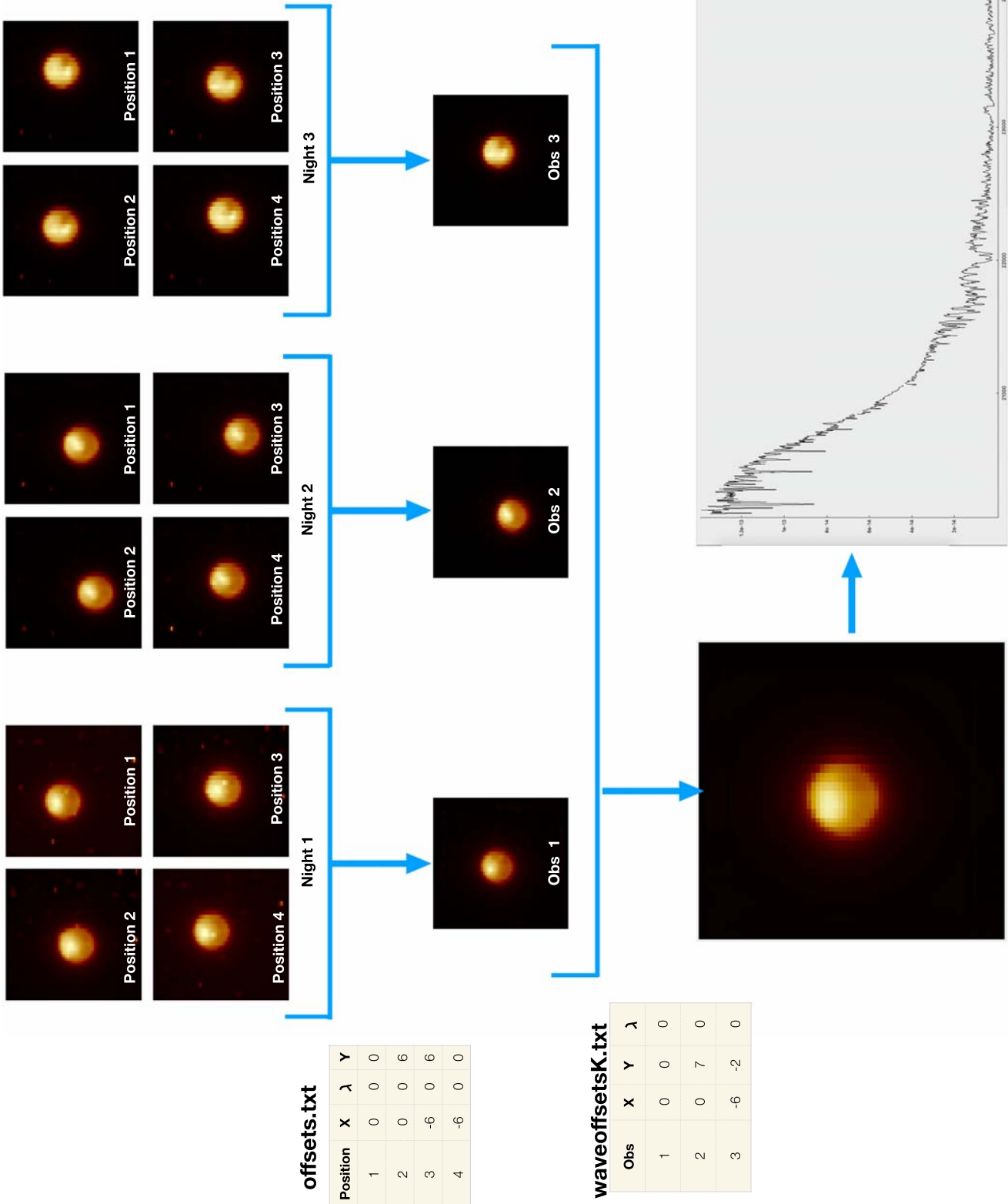

Figure 12. Example of merging sequence for NIFS data of Titan (program GN-2014A-Q-85; obtained in 2014 April/May). For each observation number, taken on a

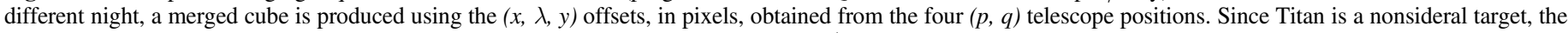

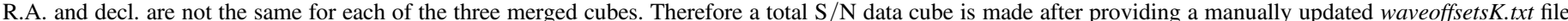

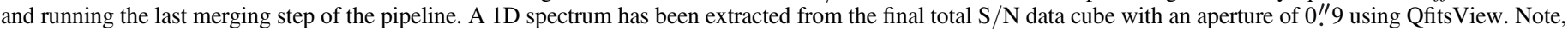
that this figure is for illustration purpose only, and depending of the science it may not make sense to merge data cubes obtained on different nights.

Titan's coordinates are not the same between merged cubes, it is not possible to just use $(p, q)$ offsets to combine the merged science 3D data cubes from different nights into a final total S/ $\mathrm{N}$ science data cube. It is a good example when the user should update manually the waveoffsetsK.txt file. To reduce Titan's NIFS data: (1) the pipeline is ran once, using the configuration file from Figure 13, and produced offsets files (e.g., offsets.txt) for each night of observation, as well as the waveoffsetsK.txt file, merged cubes for each night as well as the final full $\mathrm{S} / \mathrm{N}$ cube; (2) only the wavelength offsets should be correct in the waveoffsetsK.txt file, thus the correct $x$ and $y$ offsets are computed manually for each merged cube on different nights and the waveoffsetsK.txt file is updated; and (3) the pipeline is ran one more time to perform only the last merging task, using the updated waveoffsetsK.txt file with iraf.imcombine to create the correct full S/N 3D science cube.

\subsection{Tutorials and Examples}

The online documentation contains various examples in order to illustrate the flexibility of Nifty4Gemini and its associated NIFS PyRAF/python based pipeline. The online documentation contains multiple examples of config.cfg configuration files for science programs which can be accessed from the Gemini public archive. ${ }^{14}$ Figure 5 shows the config.

\footnotetext{
14 https://nifty4gemini.readthedocs.io/en/latest/nifty/introduction. html\#data-reduction-examples
} 


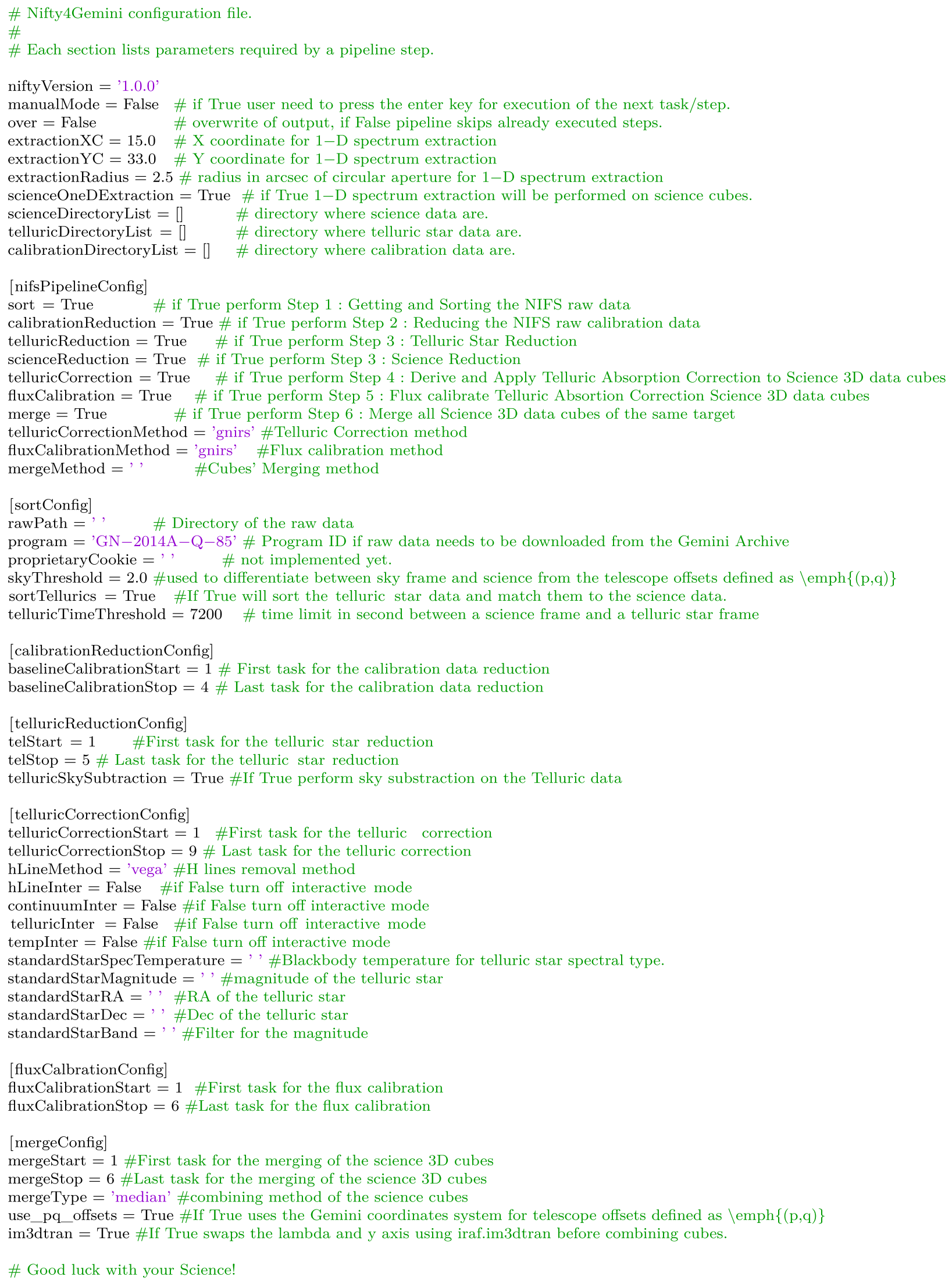

Figure 13. Example of the config.cfg configuration file used to reduce the NIFS raw data on Titan, obtained by program GN-2014A-Q-85.

cfg configuration file used to reduce the NIFS raw data on Titan, obtained by program GN-2014A-Q-85. There is also an example of the config.cfg configuration file for which the sorting of the raw data is done manually and the user specifies the various paths to the different types of data. This especially relevant when data is taken on multiple days for the same 
observation ID in the Gemini Observing Tool (OT). The config.cfg configuration file is also provided for some tutorial data that can be downloaded directly from the NIFS web page. ${ }^{15}$ In addition to the example of the config.cfg configuration files, we provide also examples of directory structures after running each of the steps of the data reduction process. ${ }^{16}$ The purpose is to help the user to identify quickly the important outputs of each step and to be able to assess if something is missing. Finally the online documentation contains various tutorials. ${ }^{17}$ The purpose is to teach the users how to run some of the tasks interactively (e.g., intrinsic star absorption removal), as well as how to incorporate their own reduced data to the pipeline (e.g., provide their own continuum-normalized telluric spectrum for telluric correction).

\section{Conclusion and Future Outlook}

In this paper, we presented an overview of the current state of Nifty4Gemini, a python package which includes a data processing pipeline for NIFS observations. The NIFS pipeline incorporates all steps needed to produce a $3 \mathrm{D}$ data cube with the total $\mathrm{S} / \mathrm{N}$ from all the acquired science exposures for a given NIFS grating configuration of the same science target. Nifty4Gemini is under continuous development. The online documentation lists the current known issues. Some of them are permanent limitations (e.g., IRAF task parameters must be 99 characters or less) while others can be addressed by further work (current telluric correction not built to be run automatically). There are plans to address the latter in a future release. In addition, future small and large possible extensions of Nifty4Gemini and its associated PyRAF/python based pipelines are described under the "future work" section of the online documentation. For instance, one extension is to have Nifty4Gemini's NIFS pipeline run automatically and internally inside the Gemini Observatory to reduce priority data and have them made available to the PI of NIFS programs the next day after the observations were taken. Another planned major extension is to incorporate to Nifty4Gemini the XDGNIRS python pipeline for GNIRS cross-disperser mode. Finally, any maintenance of the NIFS instrument that can affect the data reduction or changes of the Gemini IRAF package and AstroConda should trigger a new update of the package.

This work was made possible by the countless programmers of the Python programming language. The authors wish to acknowledge Sphinx and ReadTheDocs for building and hosting documentation, ConfigObj for providing configuration file parsing, and Github for providing version-control services. AstroConda and PyRAF are products of the Space Telescope Science Institute, which is operated by AURA for NASA. IRAF is distributed by the National Optical Astronomy Observatory, which is operated by the Association of Universities for Research in Astronomy (AURA) under a cooperative agreement with the National Science Foundation. This work is based on observations obtained at the Gemini Observatory, which is operated by the Association of Universities for Research in Astronomy, Inc., under a cooperative agreement with the NSF on behalf of the Gemini partnership: the National Science

\footnotetext{
15 http://www.gemini.edu/sciops/instruments/nifs/NIFSTutorial.tgz

16 https://nifty4gemini.readthedocs.io/en/latest/nifty/fileIO.html

17 https://nifty4gemini.readthedocs.io/en/latest/nifty/introduction. html\#tutorials
}

Foundation (United States), the National Research Council (Canada), CONICYT (Chile), Ministerio de Ciencia, Tecnología e Innovación Productiva (Argentina), and Ministério da Ciência, Tecnologia e Inovação (Brazil). The authors wish to recognize and acknowledge the very significant cultural role and reverence that the summit of Maunakea has always had within the indigenous Hawaiian community. We are most fortunate to have the opportunity to conduct observations from this mountain. All authors were also supported by Gemini Observatory. This research made use of the SIMBAD database, operated at CDS, Strasbourg, France. This publication makes use of data products from the Two Micron All Sky Survey, which is a joint project of the University of Massachusetts and the Infrared Processing and Analysis Center/California Institute of Technology, funded by the National Aeronautics and Space Administration and the National Science Foundation. We thank Inger Jorgensen, Andrew Stephens, and Tom Geballe for useful discussions and suggestions. We are also grateful for the help provided by the referee.

Facility: Gemini:Gillett(NIFS).

Software: AstroPy (Astropy Collaboration et al. 2013), AstroConda, ConfigObj, QfitsView, NDMapper, PyRAF.

\section{Appendix Gemini IRAF Tasks}

\section{A.1. nifcube}

Overview: Create data cubes from NIFS 2D format files. NIFCUBE takes input from data output by either NSFITCOORDS or NSTRANSFORM and converts the 2D data images into data cubes that have coordinates of $x$, $y$, lambda.

Description: NIFCUBE is used to resample and combine spectra from the NIFS image slicer into 3D data cubes. It is a script wrapper around the more general task GEMCUBE. This wrapper handles details of the NIFS MEF format and various checks on the processing history. Additional details about the process of creating the data cubes may be found in the help section of the GEMCUBE task.

The input NIFS spectra must be separate spectral images from each slice of the NIFS image slicer, stored as science extensions in a multiextension FITS (MEF) file, which have been mapped to world coordinates with the NSFITCOORDS task or resampled into rectified and calibrated slice data by the NSTRANSFORM task. The input files are checked for previous processing by NSFITCOORDS and a warning is issued if this is not the case. It is an error if no suitable input data is found and a warning is issued if the output already exists.

The input MEF files are generally organized as one file per exposure with each extension corresponding to one NIFS image slice on the sky. There may be science, error, and data quality extension versions. This task currently only utilizes and maps the science extensions. Each pixel in each science extension is mapped to one or more pixels in the output cube. These mapped pixels are combined to produce the output data cube or cubes. This process allows combining many input exposures, usually with some dithering or offsets, into a single data cube or creating a single data cube for each exposure. Whether multiple input files are combined into one data cube or separate data cubes are created for each input is determined by the 
number of input and output files specified. A shortcut for naming the output data cubes applies a prefix to each input file name. This shortcut is equivalent to specifying one output file name for each input file name.

The mapping from an input pixel to an output pixel depends on the input and output having world coordinates systems (WCS). The input WCS is set by the NSFITCOORDS task. Many of the details of this WCS are described in the task's help section. The NSTRANSFORM task may resample the original sampling of the slice images into rectified slice images with a new, simpler WCS. The result of NSTRANSFORM may also be used to create data cubes though generally the results of NSFITCOORDS would be used directly to eliminate an intermediate interpolation step.

The output WCS is set either using a reference data cube or using NIFCUBE parameters and certain default behaviors. In the former case the output WCS is specified by a reference image name. The image must have a 3D WCS. Generally this might be a data cube, possibly produced previously by NIFCUBE, that one wants to match. However, it could be a template WCS header in an image without pixels.

Note that the reference WCS does not specify the output coverage but only which axes correspond to wavelength and space, their orientation, and the pixel scales. The coverage is specified by the range of data in the input and by the wmin and wmax task parameters.

If a WCS reference is not supplied then the output data cube will have a sampling specified by task parameters. The orientation of the axes is set by the waxis, saxis, and taxis parameters. The default is to place the wavelength along the third or $z$-axis, the spatial sampling of a slice along the second or $y$-axis, and the sampling across the slices along the first or $x$-axis. The output pixel spatial scales are constrained to be the same, i.e., the spatial footprint of the pixels is square even though the instrumental sampling is not. The spatial size of the pixels may be explicitly set by the sscale parameter with a value of "INDEF" selecting the highest resolution in the input data. The wavelength sampling is similarly set by the wscale parameter with a default to the highest spectral resolution in the input data. There is a special syntax to select the default scales but this forces the world coordinate to increase with a pixel coordinate, "+INDEF," or decrease a pixel coordinate, "-INDEF." As with the WCS reference method, the coverage is set by the range of the input data except that a range of wavelength may be specified if desired.

Because the output coverage is set to include all the input data, with the possible exception of limiting the wavelength range of interest, there will be output pixels along the edges which do not correspond to any input pixels. The values of these output pixels is set by the blank parameter. One is free to use this value either to produce minimal impact for later applications or to provide a special value outside the range of the input data for later identification as out of bound regions.

The logfile and verbose parameters provide for the displaying and/or recording general information documenting the effects of this task.

The drizscale parameter is described further below.
3D DRIZZLE: this section summarizes the algorithm producing the output pixel values from the input pixel values. The algorithm is an extension of the algorithm first developed for 2D resampling (Fruchter \& Hook 2002) which goes by the common name "drizzle."

Each input pixel maps to a rectangular volume (voxel) with two spatial dimensions, a dispersion dimension, and some orientation. In our case the wavelength axis is assumed to be orthogonal to the spatial axes. Though the input data is $2 \mathrm{D}$, the third dimension is implicit in the slice which has a pixel width defined by the the effective slit size.

In the drizzle algorithm the value of the input voxel is apportioned to the output voxels that it overlaps by the volume of the overlap. There may be multiple input voxels that overlap the same output voxel either from the same exposure or from multiple exposures when combining data. In either case the apportioned value from the input voxels are accumulated along with the volume fraction. The accumulated fractions are used to normalize the final output data cube value.

Note that this method preserves the input pixel values independent of the output pixel sampling. This means that it is effectively preserving the input surface brightness and not the total flux. However, by the nature of this method the sum of all the pixel values which map to the output data cube is rigorously conserved.

This method is a type of linear interpolation which, like all resampling methods, degrades the resolution to some extent. However, by treating the input pixels as if they were smaller this effect can be minimized. The drizscale parameter allows the input voxel volume to be shrunk (or expanded) by specified factors. This shrinking can be uniform by specifying a single scale value or can be applied separately for each world coordinate dimension.

Because shrinking the input pixels can leave gaps in the output cube, this only makes sense when combining multiple exposures that have been offset by a noninteger number of input pixels. It is also generally used when the output is created with finer sampling than the input. The Fruchter and Hook reference provides some guidelines and discussion about how drizzling might be used.

Note that this capability will only be useful when the input data supports WCS that incorporate information about offsets, relative orientations, and dithering used during the observations. This is not yet developed for NIFS though it will be added as soon as possible.

Important Parameters:

reference $=$ " "

A reference cube whose WCS is matched in the output cubes. This does not mean the size of the cubes will be the same, but where the output and reference cubes overlap in world coordinates they will have the same pixel sampling to allow simple combining. Note that one may use a WCS image which has only a header and no data.

waxis, saxis, taxis $=3,2,1$.

The output cube axes for wavelength, the spatial component along the slices, and the spatial component across the slices, respectively.

wmin, wmax $=\mathrm{INDEF}$. 
The minimum and maximum wavelengths, in Angstroms, for the output cubes. If INDEF is specified the range of the input spectra provide the limits.

If no WCS reference is specified the following parameters are used to define the output cube sampling.

wscale, sscale = INDEF, "0.05."

The output pixel sampling for the wavelength axis, in Angstroms per pixel, and the spatial axes, in arcseconds per pixels, respectively. Note that the two spatial axes will have the same output pixel size, that is square pixels on the sky, even though the input slice sampling is different along and across the slices. If INDEF is specified the highest resolution sampling in the input data is used. If +INDEF or -INDEF is specified then the world coordinate will increase or decrease with increasing pixel coordinate in the output cube.

drizscale $=$ " $1 . "$

The volume of the input pixels in world coordinates is scaled by this factor. The value may be a single value that applies to all threes axes or three values for the two spatial axes and the wavelength axis respectively.

blank $=0$.

Output pixel value when no input data contribute to that pixel.

\section{ORCID iDs}

Marie Lemoine-Busserolle (1) https://orcid.org/0000-00030933-0690

Collin Kielty (ib https://orcid.org/0000-0002-0352-9212

Megan E. Schwamb (ib https://orcid.org/0000-0003-

4365-1455

\section{References}

Astropy Collaboration, Robitaille, T. P., Tollerud, E. J., et al. 2013, A\&A, 558, A33

Christou, J. C., Neichel, B., Rigaut, F., et al. 2010, Proc. SPIE, 7736, 77361R Fruchter, A. S., \& Hook, R. N. 2002, PASP, 114, 144

Mason, R. E., Rodríguez-Ardila, A., Martins, L., et al. 2015, ApJS, 217, 13

McGregor, P. J., Hart, J., Conroy, P. G., et al. 2003, Proc. SPIE, 4841, 1581

Pecaut, M. J., \& Mamajek, E. E. 2013, ApJS, 208, 9

Pence, W. D., Chiappetti, L., Page, C. G., Shaw, R. A., \& Stobie, E. 2010 A\&A, 524, A42

Richardson, E. H., Fletcher, J. M., Morbey, C. L., Oschmann, J. M., \& Pazder, J. S. 1998, Proc. SPIE, 3353, 600

Skrutskie, M. F., Cutri, R. M., Stiening, R., et al. 2006, AJ, 131, 1163

Wenger, M., Ochsenbein, F., Egret, D., et al. 2000, A\&AS, 143, 9

Whiting, M. T. 2012, MNRAS, 421, 3242 